\title{
Cyclic Coupled Fixed Point Result Using Kannan Type Contractions
}

\author{
Binayak S. Choudhury and Pranati Maity \\ Department of Mathematics, Bengal Engineering and Science University, Shibpur, Howrah, West Bengal 711103, India \\ Correspondence should be addressed to Pranati Maity; pranati.math@gmail.com
}

Received 17 January 2014; Revised 9 June 2014; Accepted 9 June 2014; Published 2 July 2014

Academic Editor: Aref Jeribi

Copyright (C) 2014 B. S. Choudhury and P. Maity. This is an open access article distributed under the Creative Commons Attribution License, which permits unrestricted use, distribution, and reproduction in any medium, provided the original work is properly cited.

Putting several existing ideas together, in this paper we define the concept of cyclic coupled Kannan type contraction. We establish a strong coupled fixed point theorem for such mappings. The theorem is supported with an illustrative example.

\section{Introduction and Mathematical Preliminaries}

In this paper, we establish a strong coupled fixed point result by using cyclic coupled Kannan type contractions. The following are two of several reasons why Kannan type mappings feature prominently in metric fixed point theory. They are a class of contractive mappings which are different from Banach contraction and have unique fixed points in complete metric spaces. Unlike the Banach condition, they may be discontinuous functions. Following their appearance in $[1,2]$, many persons created contractive conditions not requiring continuity of the mappings and established fixed points results of such mappings. Today, this line of research has a vast literature. Another reason for the importance of the Kannan type mapping is that it characterizes completeness which the Banach contraction does not. It has been shown in $[3,4]$, the necessary existence of fixed points for Kannan type mappings implies that the corresponding metric space is complete. The same is not true with the Banach contractions. In fact, there is an example of an incomplete metric space where every contraction has a fixed point [5]. Kannan type mappings, its generalizations, and extensions in various spaces have been considered in a large number of works some of which are in [6-10] and in references therein.

A mapping $f: X \rightarrow X$, where $(X, d)$ is a metric space, is called a Kannan type mapping if

$$
d(f x, f y) \leq k[d(x, f x)+d(y, f y)]
$$

for some $0<k<1 / 2($ see $[1,2])$.
Let $A$ and $B$ be two nonempty subsets of a set $X$. A mapping $f: X \rightarrow X$ is cyclic (with respect to $A$ and $B$ ) if $f(A) \subseteq B$ and $f(B) \subseteq A$.

The fixed point theory of cyclic contractive mappings has a recent origin. Kirk et al. [11] in 2003 initiated this line of research. This work has been followed by works like those in [12-15]. Cyclic contractive mappings are mappings of which the contraction condition is only satisfied between any two points $x$ and $y$ with $x \in A$ and $y \in B$.

The above notion of cyclic mapping is extended to the cases of mappings from $X \times X$ to $X$ in the following definition.

Definition 1. Let $A$ and $B$ be two nonempty subsets of a given set $X$. We call any function $F: X \times X \rightarrow X$ such that $F(x, y) \in B$ if $x \in A$ and $y \in B$ and $F(x, y) \in A$ if $x \in B$ and $y \in A$ a cyclic mapping with respect to $A$ and $B$.

Coupled fixed point problems have a large share in the recent development of the fixed point theory. Some examples of these works are in [16-22] and references therein. The definition of the coupled fixed point is the following.

Definition 2 (coupled fixed point [20]). An element $(x, y) \in$ $X \times X$, where $X$ is any nonempty set, is called a coupled fixed point of the mapping $F: X \times X \rightarrow X$ if $F(x, y)=x$ and $F(y, x)=y$. 
Definition 3 (strong coupled fixed point). We call the coupled fixed point in the above definition to be strong coupled fixed point if $x=y$, that is, if $F(x, x)=x$.

Combining the above concepts we define a cyclic coupled Kannan type contraction.

Definition 4 (cyclic coupled Kannan type contraction). Let $A$ and $B$ be two nonempty subsets of a metric space $(X, d)$. We call a mapping $F: X \times X \rightarrow X$ a cyclic coupled Kannan type contraction with respect to $A$ and $B$ if $F$ is cyclic with respect to $A$ and $B$ satisfying, for some $k \in(0,1 / 2)$, the inequality

$$
d(F(x, y), F(u, v)) \leq k[d(x, F(x, y))+d(u, F(u, v))],
$$

where $x, v \in A, y, u \in B$.

In this paper we introduce a coupled cyclic mapping, in particular, the cyclic coupled Kannan type contraction, and show that such mappings have strong coupled fixed points. The main result is supported with an illustrative example.

There is another standpoint from which the coupled fixed point problems can be studied, that is, as fixed point problems on product spaces [17]. In this paper we do not adopt this viewpoint. This is because this standpoint is not always helpful; in particular, there is no easy way to translate inequality (2) to another inequality in that formalism.

\section{Main Result}

Theorem 5. Let $A$ and $B$ be two nonempty closed subsets of $a$ complete metric space $(X, d)$. Let $F: X \times X \rightarrow X$ be a cyclic coupled Kannan type contraction with respect to $A$ and $B$ and $A \cap B \neq \phi$. Then $F$ has a strong coupled fixed point in $A \cap B$.

Proof. Let $x_{0} \in A$ and $y_{0} \in B$ be any two elements and let the sequences $\left\{x_{n}\right\}$ and $\left\{y_{n}\right\}$ be defined as

$$
x_{n+1}=F\left(y_{n}, x_{n}\right), \quad y_{n+1}=F\left(x_{n}, y_{n}\right) \quad \forall n \geq 0 .
$$

Then, for all $n \geq 0, x_{n} \in A$ and $y_{n} \in B$.

By (2), we have

$$
\begin{aligned}
d\left(x_{1}, y_{2}\right) & =d\left(x_{1}, F\left(x_{1}, y_{1}\right)\right) \\
& =d\left(F\left(y_{0}, x_{0}\right), F\left(x_{1}, y_{1}\right)\right) \\
& \leq k\left[d\left(y_{0}, F\left(y_{0}, x_{0}\right)\right)+d\left(x_{1}, F\left(x_{1}, y_{1}\right)\right)\right] \\
& =k\left[d\left(y_{0}, x_{1}\right)+d\left(x_{1}, y_{2}\right)\right],
\end{aligned}
$$

which implies that

$$
d\left(x_{1}, y_{2}\right) \leq t d\left(y_{0}, x_{1}\right)
$$

where

$$
0<t=\frac{k}{1-k}<1
$$

and also by (2), we have

$$
\begin{aligned}
d\left(y_{1}, x_{2}\right) & =d\left(y_{1}, F\left(y_{1}, x_{1}\right)\right) \\
& =d\left(F\left(x_{0}, y_{0}\right), F\left(y_{1}, x_{1}\right)\right) \\
& \leq k\left[d\left(x_{0}, F\left(x_{0}, y_{0}\right)\right)+d\left(y_{1}, F\left(y_{1}, x_{1}\right)\right)\right] \\
& =k\left[d\left(x_{0}, y_{1}\right)+d\left(y_{1}, x_{2}\right)\right],
\end{aligned}
$$

which implies that

$$
d\left(y_{1}, x_{2}\right) \leq t d\left(x_{0}, y_{1}\right) \text {, where } t \text { is the same as in (6). }
$$

Again, by (2), we have

$$
\begin{aligned}
d\left(x_{2}, y_{3}\right) & =d\left(x_{2}, F\left(x_{2}, y_{2}\right)\right) \\
& =d\left(F\left(y_{1}, x_{1}\right), F\left(x_{2}, y_{2}\right)\right) \\
& \leq k\left[d\left(y_{1}, F\left(y_{1}, x_{1}\right)\right)+d\left(x_{2}, F\left(x_{2}, y_{2}\right)\right)\right] \\
& =k\left[d\left(y_{1}, x_{2}\right)+d\left(x_{2}, y_{3}\right)\right]
\end{aligned}
$$

or

$$
d\left(x_{2}, y_{3}\right) \leq \frac{k}{1-k} d\left(y_{1}, x_{2}\right)
$$

which, by (8), implies that

$$
d\left(x_{2}, y_{3}\right) \leq t^{2} d\left(x_{0}, y_{1}\right),
$$

where $t$ is the same as in (6).

Similarly, by (2), we have

$$
\begin{aligned}
d\left(y_{2}, x_{3}\right) & =d\left(y_{2}, F\left(y_{2}, x_{2}\right)\right) \\
& =d\left(F\left(x_{1}, y_{1}\right), F\left(y_{2}, x_{2}\right)\right) \\
& \leq k\left[d\left(x_{1}, F\left(x_{1}, y_{1}\right)\right)+d\left(y_{2}, F\left(y_{2}, x_{2}\right)\right)\right] \\
& =k\left[d\left(x_{1}, y_{2}\right)+d\left(y_{2}, x_{3}\right)\right]
\end{aligned}
$$

or

$$
d\left(y_{2}, x_{3}\right) \leq \frac{k}{1-k} d\left(x_{1}, y_{2}\right)
$$

which, by (5), implies that

$$
d\left(y_{2}, x_{3}\right) \leq t^{2} d\left(y_{0}, x_{1}\right),
$$

where $t$ is the same as in (6).

Also, by (2), we have

$$
\begin{aligned}
d\left(x_{3}, y_{4}\right) & =d\left(x_{3}, F\left(x_{3}, y_{3}\right)\right) \\
& =d\left(F\left(y_{2}, x_{2}\right), F\left(x_{3}, y_{3}\right)\right) \\
& \leq k\left[d\left(y_{2}, F\left(y_{2}, x_{2}\right)\right)+d\left(x_{3}, F\left(x_{3}, y_{3}\right)\right)\right] \\
& =k\left[d\left(y_{2}, x_{3}\right)+d\left(x_{3}, y_{4}\right)\right]
\end{aligned}
$$


or by (14),

$$
d\left(x_{3}, y_{4}\right) \leq \frac{k}{1-k}\left[d\left(y_{2}, x_{3}\right)\right]=t^{3} d\left(y_{0}, x_{1}\right),
$$

where $t$ is the same as in (6).

Similarly, by (2), we have

$$
\begin{aligned}
d\left(y_{3}, x_{4}\right) & =d\left(y_{3}, F\left(y_{3}, x_{3}\right)\right) \\
& =d\left(F\left(x_{2}, y_{2}\right), F\left(y_{3}, x_{3}\right)\right) \\
& \leq k\left[d\left(x_{2}, F\left(x_{2}, y_{2}\right)\right)+d\left(y_{3}, F\left(y_{3}, x_{3}\right)\right)\right] \\
& =k\left[d\left(x_{2}, y_{3}\right)+d\left(y_{3}, x_{4}\right)\right]
\end{aligned}
$$

or by (11), implies that

$$
d\left(y_{3}, x_{4}\right) \leq \frac{k}{1-k}\left[d\left(x_{2}, y_{3}\right)\right]=t^{3} d\left(x_{0}, y_{1}\right),
$$

where $t$ is the same as in (6).

Let $m$ be any integer. We assume

$$
\begin{aligned}
& d\left(x_{n}, y_{n+1}\right)=d\left(x_{n}, F\left(x_{n}, y_{n}\right)\right) \leq t^{n} d\left(y_{0}, x_{1}\right), \\
& d\left(y_{n}, x_{n+1}\right)=d\left(y_{n}, F\left(y_{n}, x_{n}\right)\right) \leq t^{n} d\left(x_{0}, y_{1}\right)
\end{aligned}
$$

for all $n \leq m$ where $n$ is odd and

$$
\begin{aligned}
& d\left(x_{n}, y_{n+1}\right)=d\left(x_{n}, F\left(x_{n}, y_{n}\right)\right) \leq t^{n} d\left(x_{0}, y_{1}\right), \\
& d\left(y_{n}, x_{n+1}\right)=d\left(y_{n}, F\left(y_{n}, x_{n}\right)\right) \leq t^{n} d\left(y_{0}, x_{1}\right)
\end{aligned}
$$

for all $n \leq m$ where $n$ is even.

Let $m$ be even. Then, by (2) and (3), we have

$$
\begin{aligned}
d\left(x_{m+1}, y_{m+2}\right) & =d\left(x_{m+1}, F\left(x_{m+1}, y_{m+1}\right)\right) \\
& =d\left(F\left(y_{m}, x_{m}\right), F\left(x_{m+1}, y_{m+1}\right)\right) \\
& \leq k\left[d\left(y_{m}, x_{m+1}\right)+d\left(x_{m+1}, y_{m+2}\right)\right]
\end{aligned}
$$

or by (22), we have

$$
d\left(x_{m+1}, y_{m+2}\right) \leq \frac{k}{1-k}\left[t^{m} d\left(y_{0}, x_{1}\right)\right]=t^{m+1} d\left(y_{0}, x_{1}\right)
$$

(where $t$ is the same as in (6)).

Similarly, by (2) and (3), we have

$$
\begin{aligned}
d\left(y_{m+1}, x_{m+2}\right) & =d\left(y_{m+1}, F\left(y_{m+1}, x_{m+1}\right)\right) \\
& =d\left(F\left(x_{m}, y_{m}\right), F\left(y_{m+1}, x_{m+1}\right)\right) \\
& \leq k\left[d\left(x_{m}, y_{m+1}\right)+d\left(y_{m+1}, x_{m+2}\right)\right]
\end{aligned}
$$

or by (21), we have

$$
d\left(y_{m+1}, x_{m+2}\right) \leq \frac{k}{1-k}\left[t^{m} d\left(x_{0}, y_{1}\right)\right]=t^{m+1} d\left(x_{0}, y_{1}\right)
$$

(where $t$ is the same as in (6)).

Again, let $m$ be odd.
Then, by (2) and (3), we have

$$
\begin{aligned}
d\left(x_{m+1}, y_{m+2}\right) & =d\left(x_{m+1}, F\left(x_{m+1}, y_{m+1}\right)\right) \\
& =d\left(F\left(y_{m}, x_{m}\right), F\left(x_{m+1}, y_{m+1}\right)\right) \\
& \leq k\left[d\left(y_{m}, x_{m+1}\right)+d\left(x_{m+1}, y_{m+2}\right)\right]
\end{aligned}
$$

or by (20), we have

$$
d\left(x_{m+1}, y_{m+2}\right) \leq \frac{k}{1-k}\left[t^{m} d\left(x_{0}, y_{1}\right)\right]=t^{m+1} d\left(x_{0}, y_{1}\right)
$$

(where $t$ is the same as in (6)).

Similarly, by (2) and (3), we have

$$
\begin{aligned}
d\left(y_{m+1}, x_{m+2}\right) & =d\left(y_{m+1}, F\left(y_{m+1}, x_{m+1}\right)\right) \\
& =d\left(F\left(x_{m}, y_{m}\right), F\left(y_{m+1}, x_{m+1}\right)\right) \\
& \leq k\left[d\left(x_{m}, y_{m+1}\right)+d\left(y_{m+1}, x_{m+2}\right)\right]
\end{aligned}
$$

or by (19), we have

$$
d\left(y_{m+1}, x_{m+2}\right) \leq \frac{k}{1-k}\left[t^{m} d\left(y_{0}, x_{1}\right)\right]=t^{m+1} d\left(y_{0}, x_{1}\right)
$$

(where $t$ is the same as in (6)).

Thus (19)-(22) hold for $m+1$. But we have shown in (5), (8)-(18) that this is valid for $m=1,2,3$. Then, by induction, we conclude that (19)-(22) are valid for all $m$.

From the above we conclude that, for all odd integer $n$, we have

$$
\begin{aligned}
& d\left(x_{n}, y_{n+1}\right)=d\left(x_{n}, F\left(x_{n}, y_{n}\right)\right) \leq t^{n} d\left(y_{0}, x_{1}\right), \\
& d\left(y_{n}, x_{n+1}\right)=d\left(y_{n}, F\left(y_{n}, x_{n}\right)\right) \leq t^{n} d\left(x_{0}, y_{1}\right)
\end{aligned}
$$

and for all even integer $n$, we have

$$
\begin{aligned}
& d\left(x_{n}, y_{n+1}\right)=d\left(x_{n}, F\left(x_{n}, y_{n}\right)\right) \leq t^{n} d\left(x_{0}, y_{1}\right), \\
& d\left(y_{n}, x_{n+1}\right)=d\left(y_{n}, F\left(y_{n}, x_{n}\right)\right) \leq t^{n} d\left(y_{0}, x_{1}\right) .
\end{aligned}
$$

By (2), we have

$$
\begin{aligned}
d\left(x_{1}, y_{1}\right) & =d\left(F\left(y_{0}, x_{0}\right), F\left(x_{0}, y_{0}\right)\right) \\
& \leq k\left[d\left(y_{0}, F\left(y_{0}, x_{0}\right)\right)+d\left(x_{0}, F\left(x_{0}, y_{0}\right)\right)\right] \\
& =\frac{t}{t+1}\left[d\left(y_{0}, x_{1}\right)+d\left(x_{0}, y_{1}\right)\right]
\end{aligned}
$$

(where $t$ is the same as in (6)). 
Again, by (2), we have

$$
\begin{aligned}
& d\left(x_{2}, y_{2}\right) \\
& =d\left(F\left(y_{1}, x_{1}\right), F\left(x_{1}, y_{1}\right)\right) \\
& \leq k\left[d\left(y_{1}, F\left(y_{1}, x_{1}\right)\right)+d\left(x_{1}, F\left(x_{1}, y_{1}\right)\right)\right] \\
& =k\left[d\left(y_{1}, x_{2}\right)+d\left(x_{1}, y_{2}\right)\right] \\
& \leq k\left[t d\left(x_{0}, y_{1}\right)+t d\left(y_{0}, x_{1}\right)\right] \quad(\text { by }(5) \text { and }(8)) \\
& =\frac{t^{2}}{t+1}\left[d\left(y_{0}, x_{1}\right)+d\left(x_{0}, y_{1}\right)\right]
\end{aligned}
$$

(where $t$ is the same as in (6)) .

Let, for some integer $m$,

$$
d\left(x_{m}, y_{m}\right) \leq \frac{t^{m}}{t+1}\left[d\left(x_{0}, y_{1}\right)+d\left(y_{0}, x_{1}\right)\right]
$$

Let $m$ be odd. Then, by (2) and (3), we have

$$
\begin{aligned}
& d\left(x_{m+1}, y_{m+1}\right) \\
& =d\left(F\left(y_{m}, x_{m}\right), F\left(x_{m}, y_{m}\right)\right) \\
& \leq k\left[d\left(y_{m}, x_{m+1}\right)+d\left(x_{m}, y_{m+1}\right)\right] \\
& \leq k\left[t^{m}\left[d\left(x_{0}, y_{1}\right)+d\left(y_{0}, x_{1}\right)\right]\right] \quad(\text { by }(31)) \\
& =\frac{t^{m+1}}{t+1}\left[d\left(x_{0}, y_{1}\right)+d\left(y_{0}, x_{1}\right)\right] \quad(\text { by }(6)) .
\end{aligned}
$$

Again, let $m$ be even. Then, by (2) and (3), we have

$$
\begin{aligned}
d\left(x_{m+1}, y_{m+1}\right) & \\
& =d\left(F\left(y_{m}, x_{m}\right), F\left(x_{m}, y_{m}\right)\right) \\
& \leq k\left[d\left(y_{m}, x_{m+1}\right)+d\left(x_{m}, y_{m+1}\right)\right] \\
& \leq k\left[t^{m}\left[d\left(y_{0}, x_{1}\right)+d\left(x_{0}, y_{1}\right)\right]\right] \quad(\text { by }(32)) \\
& =\frac{t^{m+1}}{t+1}\left[d\left(y_{0}, x_{1}\right)+d\left(x_{0}, y_{1}\right)\right] \quad(\text { by }(6)) .
\end{aligned}
$$

Therefore, (35) also holds if we replace $m$ by $m+1$. But, (33) and (34) imply that (35) is true for $m=1,2$.

Then, by induction, for all $n$, it follows that

$$
d\left(x_{n}, y_{n}\right) \leq \frac{t^{n}}{t+1}\left[d\left(x_{0}, y_{1}\right)+d\left(y_{0}, x_{1}\right)\right] \text {. }
$$

Now, by (31)-(32) and (38), we have

$$
\begin{aligned}
& d\left(x_{n}, x_{n+1}\right)+d\left(y_{n}, y_{n+1}\right) \\
& \leq d\left(x_{n}, y_{n}\right)+d\left(y_{n}, x_{n+1}\right)+d\left(y_{n}, x_{n}\right)+d\left(x_{n}, y_{n+1}\right) \\
&= {\left[d\left(x_{n}, y_{n}\right)+d\left(y_{n}, x_{n}\right)\right]+\left[d\left(y_{n}, x_{n+1}\right)+d\left(x_{n}, y_{n+1}\right)\right] } \\
& \leq \frac{2 t^{n}}{t+1}\left[d\left(x_{0}, y_{1}\right)+d\left(y_{0}, x_{1}\right)\right] \\
&+t^{n}\left[d\left(x_{0}, y_{1}\right)+d\left(y_{0}, x_{1}\right)\right] .
\end{aligned}
$$

Since $0<t<1$, it follows that $\sum d\left(x_{n}, x_{n+1}\right)+\sum d\left(y_{n}, y_{n+1}\right)<$ $\infty$. This implies that $\left\{x_{n}\right\}$ and $\left\{y_{n}\right\}$ are Cauchy sequences and hence are convergent.

Since $A$ and $B$ are closed subsets, $\left\{x_{n}\right\} \subset A$, and $\left\{y_{n}\right\} \subset B$, it follows that

$$
x_{n} \longrightarrow x \in A, \quad y_{n} \longrightarrow y \in B \quad \text { as } n \longrightarrow \infty .
$$

Again, from (35), $d\left(x_{n}, y_{n}\right) \rightarrow 0$ as $n \rightarrow \infty$.

Therefore, from (38),

$$
x=y \text {. }
$$

Since $A \cap B \neq \phi$, then from the above it follows that $x \in A \cap B$.

Now, by (2) and (3), we have

$$
\begin{aligned}
& d(x, F(x, y)) \\
& \quad \leq d\left(x, x_{n+1}\right)+d\left(x_{n+1}, F(x, y)\right) \\
& \quad=d\left(x, x_{n+1}\right)+d\left(F\left(y_{n}, x_{n}\right), F(x, y)\right) \\
& \quad \leq d\left(x, x_{n+1}\right)+k\left[d\left(y_{n}, x_{n+1}\right)+d(x, F(x, y))\right]
\end{aligned}
$$

or

$$
d(x, F(x, y)) \leq \frac{1}{1-k} d\left(x, x_{n+1}\right)+\frac{k}{1-k} d\left(y_{n}, x_{n+1}\right) .
$$

Taking the limit as $n \rightarrow \infty$ in the above inequality, using (40) and (41), we obtain $d(x, F(x, y))=0$. Again, in view of (41), we conclude that $x=F(x, x)$; that is, we have a strong coupled fixed point of $F$.

This completes the proof of the theorem.

Example 6. Let $X=R$ with the metric defined as $d(x, y)=$ $|x-y|$.

Let $A=[-\pi, 0]$ and $B=[0, \pi]$.

Then $A$ and $B$ are nonempty closed subsets of $X$ and $d(A, B)=0$.

Let $F: X \times X \rightarrow X$ be defined as

$$
\begin{aligned}
& F(\alpha, \beta) \\
& = \begin{cases}\frac{-\operatorname{sgn}(\alpha)}{3}\left|\alpha \sin \frac{1}{\alpha}\right|, & \text { if }(\alpha, \beta) \in A \times B, \\
+\frac{\operatorname{sgn}(\beta)}{3}\left|\beta \sin \frac{1}{\beta}\right|, & \text { if }(\alpha, \beta) \in B \times A, \\
2, & \text { if }(\alpha, \beta) \notin(A \times B) \cup(B \times A) .\end{cases}
\end{aligned}
$$

Let $k=1 / 3$.

Then $d(F(x, y), F(u, v))=(1 / 3)|x \sin (1 / x)+u \sin (1 / u)|$. 
And

$$
\begin{aligned}
k & {[d(x, F(x, y))+d(u, F(u, v))] } \\
& =\frac{1}{3}[d(x, F(x, y))+d(u, F(u, v))] \\
& =\frac{1}{3}\left[\left|x+\frac{1}{3} x \sin \frac{1}{x}\right|+\left|u-\frac{1}{3} u \sin \frac{1}{u}\right|\right] \\
& \leq \frac{1}{3}\left[\left|x+\frac{1}{3} x \sin \frac{1}{x}\right|+\left|u+\frac{1}{3} u \sin \frac{1}{u}\right|\right] \\
& =\frac{1}{3}\left[|x+u|+\frac{1}{3}\left|x \sin \frac{1}{x}+u \sin \frac{1}{u}\right|\right] .
\end{aligned}
$$

From the above we see that the inequality (2) is satisfied. Thus all the conditions of the Theorem 5 are satisfied. By an application of Theorem 5 , there is a strong coupled fixed point of $F$. Here $(0,0)$ is a strong coupled fixed point of $F$; that is, $F(0,0)=0$.

Remark 7. The example considered here is truly a cyclic coupled Kannan type. This can be seen by choosing $x=0, y=$ $-1, v=-1 / \pi$, all belonging to $A$ and $u=1$, belonging to $B$, and observing that inequality (2) is not satisfied. Also $F$ is not continuous.

\section{Conflict of Interests}

The authors declare that there is no conflict of interests regarding the publication of this paper.

\section{References}

[1] R. Kannan, "Some results on fixed points," Bulletin of the Calcutta Mathematical Society, vol. 60, pp. 71-76, 1968.

[2] R. Kannan, "Some results on fixed points II," The American Mathematical Monthly, vol. 76, pp. 405-408, 1969.

[3] N. Shioji, T. Suzuki, and W. Takahashi, "Contractive mappings, Kannan mappings and metric completeness," Proceedings of the American Mathematical Society, vol. 126, no. 10, pp. 3117-3124, 1998.

[4] P. V. Subrahmanyam, "Completeness and fixed-points," Monatshefte für Mathematik, vol. 80, no. 4, pp. 325-330, 1975.

[5] E. H. Connell, "Properties of fixed point spaces," Proceedings of the American Mathematical Society, vol. 10, pp. 974-979, 1959.

[6] D. Ariza-Ruiz and A. Jimnez-Melado, "A continuation method for weakly kannan maps," Fixed Point Theory and Applications, vol. 2010, Article ID 321594, 12 pages, 2010.

[7] B. S. Choudhury and K. Das, "Fixed points of generalized Kannan type mappings in generalized Menger spaces," Korean Mathematical Society. Communications, vol. 24, no. 4, pp. 529537, 2009.

[8] B. S. Choudhury and A. Kundu, "A Kannan-like contraction in partially ordered spaces," Demonstratio Mathematica, vol. 46, no. 2, pp. 327-334, 2013.

[9] B. Damjanović and D. Dragan, "Multivalued generalizations of the Kannan fixed point theorem," Filomat, vol. 25, no. 1, pp. 125131, 2011.

[10] S. Moradi and D. Alimohammadi, "New extensions of Kannan fixed-point theorem on complete metric and generalized metric spaces," International Journal of Mathematical Analysis, vol. 5, no. 45-48, pp. 2313-2320, 2011.

[11] W. A. Kirk, P. S. Srinivasan, and P. Veeramani, "Fixed points for mappings satisfying cyclical contractive conditions," Fixed Point Theory, vol. 4, no. 1, pp. 79-89, 2003.

[12] B. S. Choudhury, K. Das, and S. K. Bhandari, "A generalized cyclic $C$-contraction principle in Menger spaces using a control function," International Journal of Applied Mathematics, vol. 24, no. 5, pp. 663-673, 2011.

[13] E. Karapınar, "Fixed point theory for cyclic weak $\phi$ contraction," Applied Mathematics Letters, vol. 24, no. 6, pp. 822-825, 2011.

[14] I. A. Rus, "Cyclic representations and fixed points," Annals of the Tiberiu Popoviciu Seminar of Functional Equations, Approximation and Convexity, vol. 3, pp. 171-178, 2005.

[15] M. Păcurar and I. A. Rus, "Fixed point theory for cyclic $\varphi$ contractions," Nonlinear Analysis. Theory, Methods \& Applications A, vol. 72, no. 3-4, pp. 1181-1187, 2010.

[16] V. Berinde, "Generalized coupled fixed point theorems for mixed monotone mappings in partially ordered metric spaces," Nonlinear Analysis. Theory, Methods \& Applications A, vol. 74, no. 18, pp. 7347-7355, 2011.

[17] V. Berinde, "Coupled fixed point theorems for $\phi$-contractive mixed monotone mappings in partially ordered metric spaces," Nonlinear Analysis. Theory, Methods \& Applications A, vol. 75, no. 6, pp. 3218-3228, 2012.

[18] B. S. Choudhury and A. Kundu, "A coupled coincidence point result in partially ordered metric spaces for compatible mappings," Nonlinear Analysis. Theory, Methods \& Applications A, vol. 73, no. 8, pp. 2524-2531, 2010.

[19] B. S. Choudhury and P. Maity, "Coupled fixed point results in generalized metric spaces," Mathematical and Computer Modelling, vol. 54, no. 1-2, pp. 73-79, 2011.

[20] T. Gnana Bhaskar and V. Lakshmikantham, "Fixed point theorems in partially ordered metric spaces and applications," Nonlinear Analysis. Theory, Methods \& Applications A, vol. 65, no. 7, pp. 1379-1393, 2006.

[21] V. Lakshmikantham and L. Ćirić, "Coupled fixed point theorems for nonlinear contractions in partially ordered metric spaces," Nonlinear Analysis. Theory, Methods \& Applications A, vol. 70, no. 12, pp. 4341-4349, 2009.

[22] B. Samet and C. Vetro, "Coupled fixed point theorems for multivalued nonlinear contraction mappings in partially ordered metric spaces," Nonlinear Analysis. Theory, Methods \& Applications A, vol. 74, no. 12, pp. 4260-4268, 2011. 


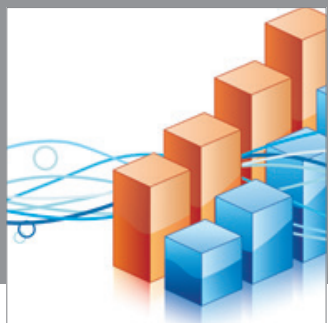

Advances in

Operations Research

mansans

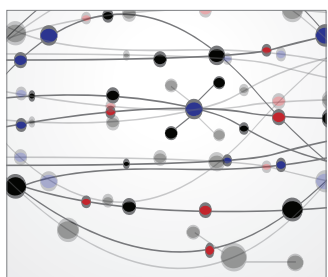

The Scientific World Journal
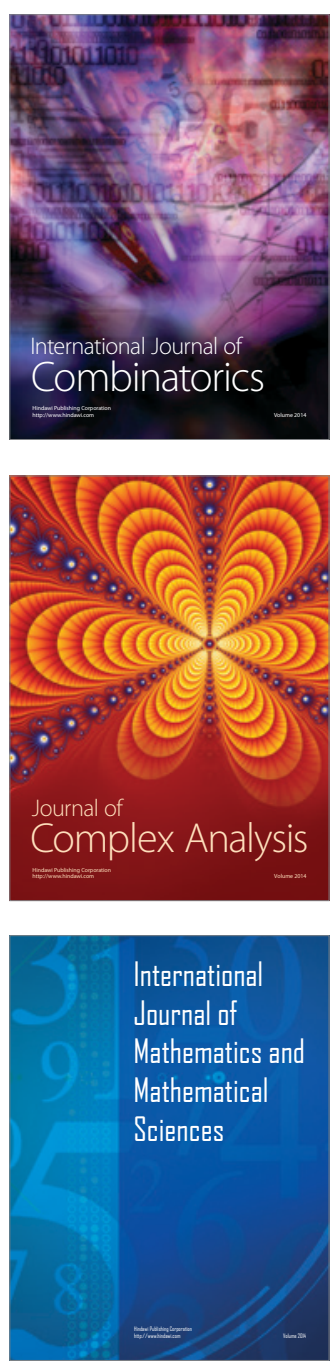
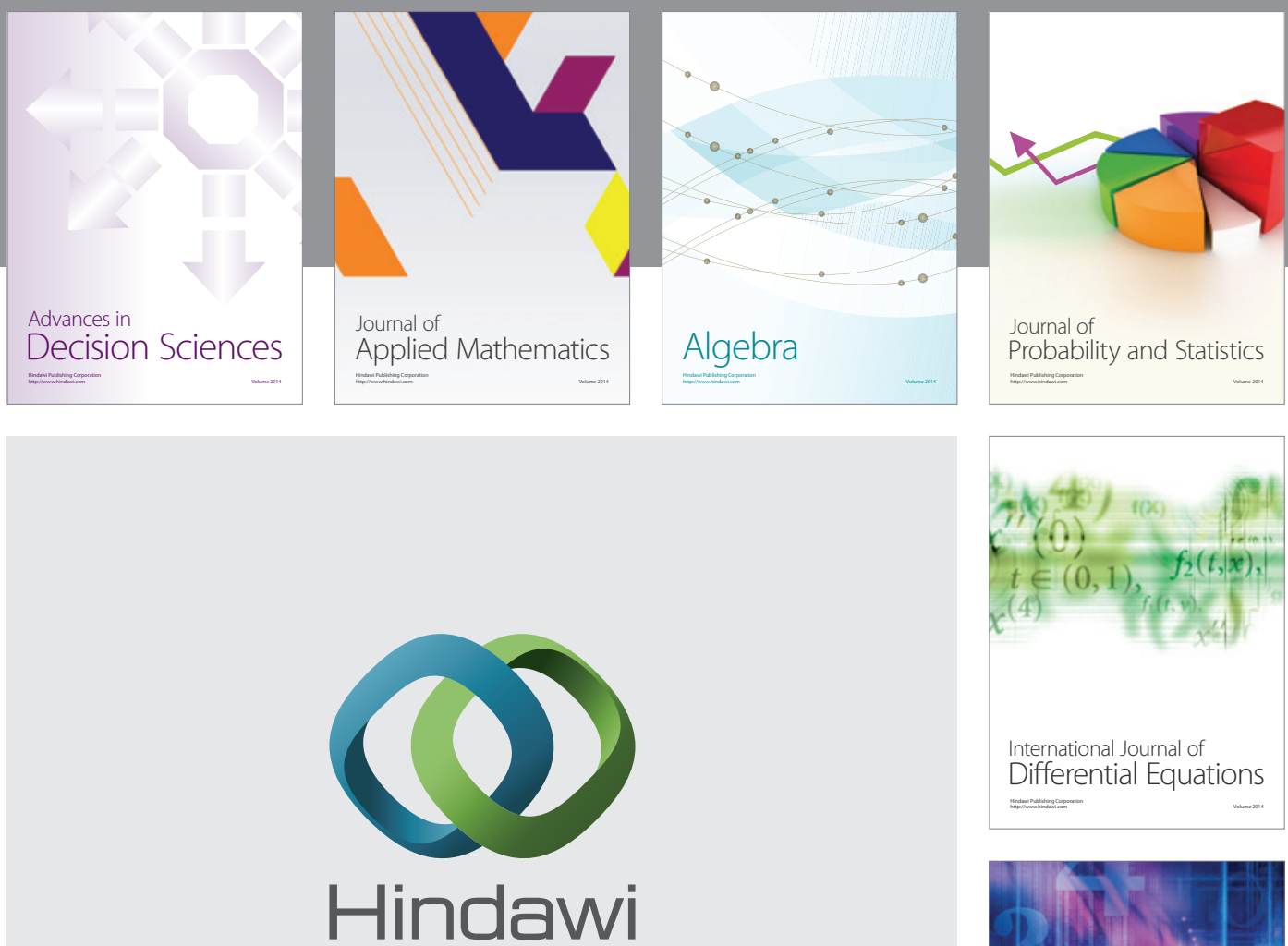

Submit your manuscripts at http://www.hindawi.com
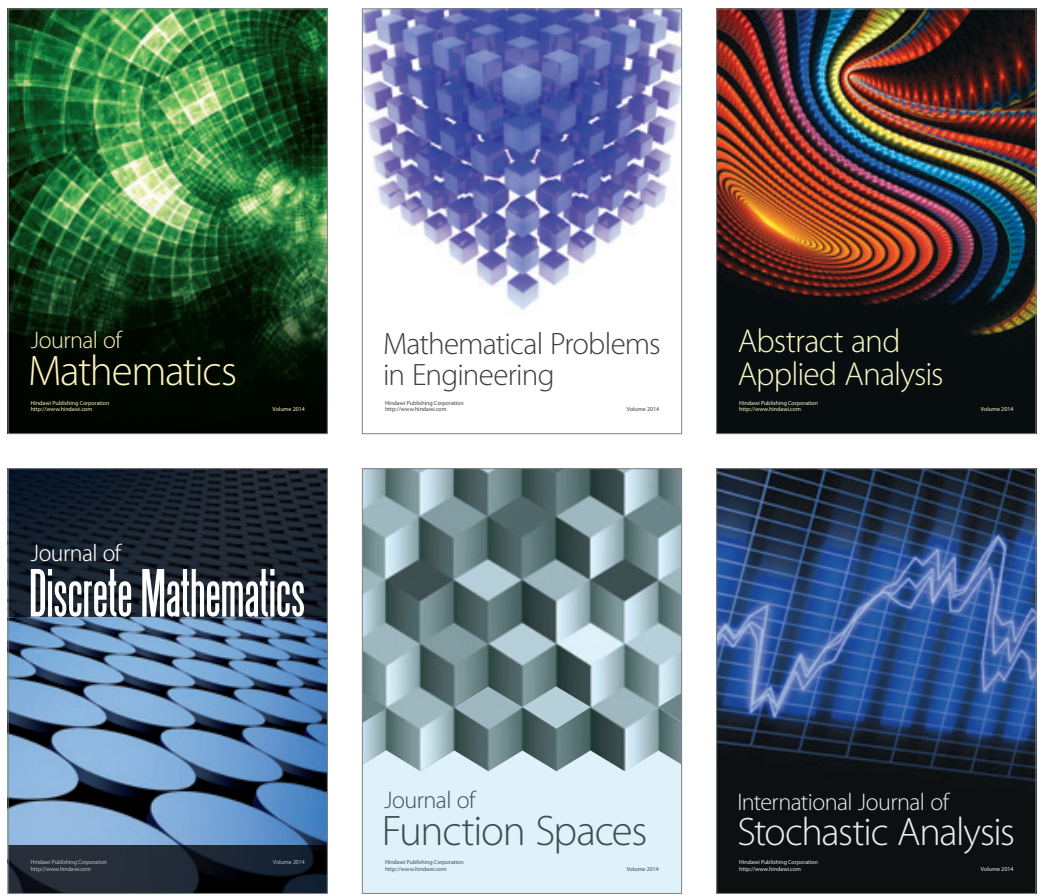

Journal of

Function Spaces

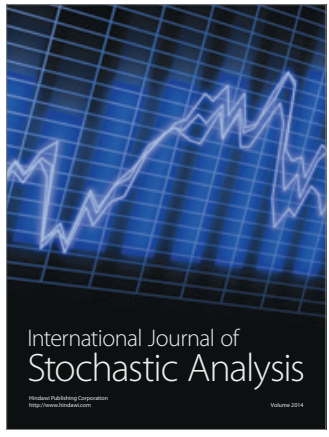

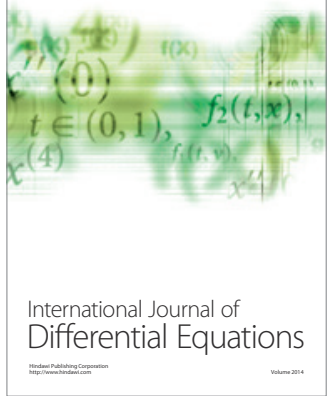
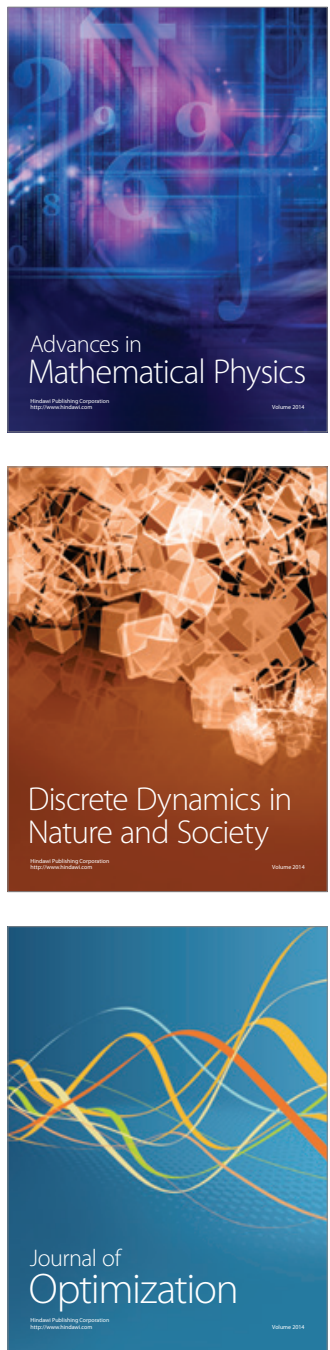